\title{
ÉDITORIAL
}

FRANCIS GENDREAU

\section{La conférence du Caire : les intégrismes contre le développement}

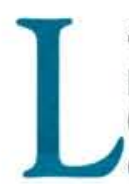

a Conférence internationale sur la population et le développement (Le Caire, 5 au 13 septembre 1994) était la troisième conférence réunie sur ces questions à un niveau politique, après celles de Bucame (1974) et de Mexico (1984). Les 180 pays représentés ont adopté, après huit jours de débats intenses, un "Programme d'action" qui fixe à la communauté internationale des objectifs dans le domaine de la population, notamment en termes d'éducation, de réduction de la mortalité et d'accès à la planification familiale. En attendant de revenir plus longuement dans une prochaine livraison sur toutes ces questions, nous retiendrons ici trois idéesforces de cette manifestation.

Dans le contexte mondial actuel, les débats n'ont pas été perturbés par des affrontements Est-Ouest ou Nord-Sud, ni par des questions très présentes dans les enceintes internationales dans les années récentes, comme les territoires occupés ou l'apartheid. Mais ils ont été dominés par l'irruption en force du religieux dans la sphère démographique (jamais manifestation de ce type n'aura autant mérité d'être qualifiée de "grand-messe" !). Les échanges ont été rudes sur de nombreux points: l'égalité entre hommes et femmes, les différentes formes du mariage et de la famille, le concept de "santé sexuelle", les droits en matière de sexualité et de procréation, l'avortement (à ou sans risque, légal ou non). En effet, face à une approche laïque et relativement ouverte de ces questions, une opposition bruyante s'est manifestée, résultant de la convergence de deux courants reli- gieux fortement teintés d'intégrisme : le Saint-Siège d'une part, appuyé par plusieurs pays d'Amérique latine, certains pays musulmans d'autre part. Finalement, le Programme d'action a été approuvé par consensus, avec des réserves de certains de ces pays sur différentes parties du texte, cela malgré la référence aux valeurs religieuses qui y avait été introduite à leur demande. L'esprit du document en a été profondément modifié et son caractère laïc sérieusement altéré. Deux questions méritent sans doute d'être posées: n'est-il vraiment pas possible d'aborder sereinement en cette fin de siècle ces problèmes importants au plan de l'éthique individuelle et collective? La société civile ne peut-elle pas se positionner plus clairement par rapport à la sphère religieuse?

Second constat, l'absence de véritable débat sur la question du développement. Cette absence est d'ailleurs en partie due à la focalisation des débats sur les points évoqués ci-dessus. Deux avancées sont toutefois à noter en ce domaine : la reconnaissance du "droit au développement", "droit universel et inaliénable faisant intégralement partie des droits fondamentaux de l'être humain » et une conception des politiques de population allant très au-delà de la seule planification familiale, puisque y incluant notamment les actions en matière d'éducation et de " renforcement du pouvoir des femmes ". Mais le problème des relations entre la population, l'environnement et le développement a été curieusement évacué des discussions malgré le titre officiel de la Conférence. L'incantation sur " les liens entre population, croissance économique soutenue et développement durable " est renouvelée, mais non explicitée. L'esprit du texte reste très néomalthusien alors que la recherche n'a guère progressé sur l'importance, voire même le sens de ces relations.

On peut enfin noter, parmi les nouveautés que comporte le Programme d'action par rapport aux textes antérieurs, l'objectif de stabilisation de la population mondiale que s'est fixé la communauté internationale : il y a là un élément tout à fait nouveau si l'on se rappelle les discours sur "l'explosion démographique " encore dominants jusqu'à une période récente. Aujourd'hui, c'est plutôt de relaxation démographique qu'il faut parler. En effet, les statistiques montrent bien la baisse de la fécondité au niveau mondial : le niveau mondial moyen de la fécondité était estimé à 4,9 enfants par femme en 1965-70; il est estimé à 3,1 en 1990-95. Le taux d'accroissement de la population mondiale, après être passé par un maximum en 1965-70 $(2,1 \%)$, décroît régulièrement depuis : il est d'environ $1,8 \%$ en 1990-95. Les Nations Unies envisagent la poursuite de cette tendance dans les décennies à venir, avec à terme (dans le courant du xxle siècle ?) une croissance nulle, c'est-à-dire une stabilisation de la population mondiale aux alentours de 11,5 milliards d'habitants (contre 5,6 aujourd'hui) : après la Conférence du Caire, ce scénario devient un objectif à atteindre. L'humanité est-elle capable de s'organiser pour absorber ce dernier doublement de son effectif ? C'est l'enjeu majeur de nos sociétés, enjeu politique, économique, social et environnemental beaucoup plus que démographique. 